\title{
Early introduction of water and complementary feeding and nutritional status of children in northern Senegal
}

\author{
Neil Gupta ${ }^{1}$, Mario Gehri ${ }^{2}$ and Nicolas Stettler ${ }^{3,4, *}$ \\ 'University of Pennsylvania School of Medicine, Philadelphia, PA, USA: ${ }^{2}$ Department of Pediatrics, Centre \\ Hospitalier Universitaire Vaudois, Lausanne, Switzerland: '3ivision of Gastroenterology, Hepatology and Nutrition, \\ North 1559, The Children's Hospital of Philadelphia, 34th Street and Civic Center Boulevard, Philadelphia, PA \\ 19104-4399, USA: ${ }^{4}$ Center for Clinical Epidemiology and Biostatistics, University of Pennsylvania School of \\ Medicine, Philadelphia, PA, USA
}

\section{Submitted 22 March 2006: Accepted 19 January 2007: First published online 15 March 2007}

\begin{abstract}
Objective: Malnutrition is responsible globally for $60 \%$ of deaths among children under 5 years and is often attributed to suboptimal feeding practices. In response, the World Health Organization recommends exclusive breast-feeding for the first 6 months of life. The objective of this study was to determine if an association exists between the early introduction of water and complementary foods (CFs) and the nutritional status of children in northern Senegal.

Design/Setting/Subjects: A cross-sectional study of 374 children in the Podor Health District between the ages of 6 and 23 months was conducted. Knowledge and behaviours of mothers regarding introduction of water and CFs were assessed via individual interviews.

Results: Water was introduced to about $85 \%$ of the children in the first 3 months of life and $62 \%$ were fed CFs before 6 months. Overall, 16\% had clinically significant wasting (weight-for-length $Z$-score (WHZ) less than - 2) and 20\% had stunting (height-for-age $Z$-score (HAZ) less than -2). There was no significant association between wasting or stunting and introduction of water before 3 months (WHZ: odds ratio $=0.99,95 \%$ confidence interval $0.46-2.14, P=0.97$; HAZ: $0.68,0.34-1.36, P=0.3$ ) or introduction of CFs before 6 months (WHZ: 0.81, 0.46-1.42, $P=0.5$; HAZ: 0.79 , $0.46-1.35, P=0.4)$. A significant association was found between wasting and male sex, age, living in Guede community, drinking river/pond water and large family size, while stunting was associated with age and drinking tap water.

Conclusion: The results of the present study suggest that early introduction of water and CFs is frequent and is not associated with increased risk for malnutrition among children from this region of northern Senegal, but the possibility of reverse causality cannot be excluded.
\end{abstract}

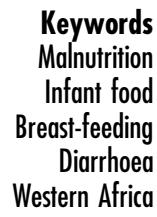

Globally, malnutrition is responsible for $60 \%$ of the 10.9 million annual deaths among children under 5 , with over two-thirds of these deaths occurring during the first year of life ${ }^{1}$. Malnutrition is associated with $61 \%$ of deaths from diarrhoeal disease $\mathrm{e}^{2,3}$ and is a major prognostic indicator for diarrhoea-related mortality and convalescence ${ }^{4}$. The World Health Organization (WHO) recommends exclusive breast-feeding for the first 6 months of life, with nutritionally adequate and safe complementary feeding while breast-feeding continues up to 2 years of life ${ }^{1}$. Exclusive breast-feeding during the first 6 months of life is recommended to avoid exposure to contaminants and displacement of breast-feeding by water or other foods. Despite the well-known advantages of breast-feeding 5,6 and WHO recommendations, $<35 \%$ of infants worldwide are exclusively breast-fed for the first 4 months of life ${ }^{1}$, and many studies have demonstrated a high prevalence of early introduction of water and complementary foods (CFs) in the African setting ${ }^{7,8}$.

The objective of this study was to determine if an association exists between the early introduction of water (before 3 months) and CFs (before 6 months) and the nutritional status of infants and young children in northern Senegal, a region where early introduction of water and CFs is a frequent practice. This study additionally aimed to assess by qualitative analysis the underlying knowledge and behaviours regarding early introduction of water and CFs in this region.

\section{Methods}

\section{Study design}

The Podor Health District, located in the rural desert region of northern Senegal, covers an area of $13000 \mathrm{~km}^{2}$ 
and is home to 186000 inhabitants, with health coverage of 4325 individuals per health post. The semi-urban town of Podor and rural communities of Guede, Gamadji and Galoya together represent $>70 \%$ of the population of the district.

Phase I of this study is based on survey data collected in the Podor Health District by Fondation Terre des Hommes (TDH) to compare health programmes funded by TDH (intervention zone) with areas without such programmes (control zone) ${ }^{9}$. Phase II of this study is a qualitative assessment of knowledge and behaviours of mothers in the region with regard to introduction of water and CFs in their infants.

\section{Phase I - quantitative analysis}

In December 2002, TDH conducted a survey of 311 households, collecting data on a total of 374 children aged 6-23 months from 20 villages, which was a subset of a larger survey of children aged 0-36 months ${ }^{9}$. Subjects were randomly selected according to previously described sampling methods ${ }^{10}$. Although the response rate was not systematically assessed, it is estimated to be $>90 \%$ based on similar surveys conducted in this region previously.

Survey questions were designed to characterise the child's environment, sociodemographics, preventive health care and recent medical history (past 15 days). The child's family was defined as the child's siblings, parents and grandparents, as well as the father's additional wives and children (half-siblings of the study subjects). Children were weighed naked to the nearest $100 \mathrm{~g}$ using a Salter-Brecknell scale. Length was measured in the recumbent position to the nearest centimetre using a wooden measuring board (method described elsewhere) $)^{11}$.

The two primary exposures were defined as introduction of water earlier than 3 months and introduction of CFs earlier than 6 months of life. Both child age and age at introduction of water and CFs were determined by maternal recall to the nearest month. For age at introduction of water and CFs, parents were asked 'Pour l'alimentation de votre enfant, quand avez vous introduit l'eau?' [For the feeding of your child, when did you introduce water?] and 'Pour l'alimentation de votre enfant, quand avez vous introduit un autre aliment (la bouillie or autre lait)?' [For the feeding of your child, when did you introduce another food (porridge or other milk)]? The French questions were translated into Pulaar by local translators.

Although the WHO recommends that water should also not be introduced before 6 months, only a small number of subjects adhered to those recommendations, precluding meaningful analyses. The primary outcome, nutritional status, was calculated as weight-for-length $Z$-scores (WHZs) and height-for-age Z-scores (HAZs) smaller than two standard deviations (SDs) below the mean with reference to 1978 CDC (Centers for Disease
Control)/WHO growth reference values using the Epi-info software program ${ }^{11,12}$. WHZ less than -2 SD is a well established indicator for wasting or thinness usually due to recent and severe weight loss, often associated with acute starvation or severe disease, while HAZ less than -2 SD indicates chronic stunting, often due to repeated exposure to adverse conditions ${ }^{12}$.

Statistical and graphical distributions of the main exposures, outcomes and confounding variables were used for descriptive analysis. The unadjusted associations of the main exposure and outcome and confounding variables were determined by $\chi^{2}$ analysis and expressed as odds ratios and 95\% confidence intervals. In secondary analyses, linear regression for these associations was conducted using WHZ and HAZ as continuous variables. Effect modifiers (interactions) were explored by stratified analyses or using an interaction term, and multiple logistic regressions were used to adjust for potentially confounding variables. All analyses were performed using the SPSS 10.0 statistical software package.

\section{Phase II - qualitative assessment}

Qualitative assessment was conducted via semi-structured individual interviews with 43 mothers, two health care workers and one religious leader in Podor Health District. These interviews were designed with open-ended questions to determine knowledge and behaviours regarding timing, source and preparation of water and food for the infant, as well as identification and management of diarrhoeal disease. Five villages were selected to represent a diversity of socioeconomic and geographic backgrounds.

Interviews were conducted with a convenience sample of 8-10 mothers who were available in proximity to the health centre in each village. The health care workers and religious leader were chosen as key informants. Behaviours reported by three or more mothers in at least two different villages were considered representative of a commonly held belief or behaviour of the greater population. Responses not meeting these criteria were considered isolated beliefs and are therefore not reported here.

All interviews were conducted by N.G. (principal investigator) with a local Pulaar translator experienced in household surveys. Informed consent was obtained verbally from all respondents prior to the interview. The study was approved by the Ethics Committee of the Faculty of Medicine of Lausanne and the Institutional Review Board of the University of Pennsylvania.

\section{Results}

A total of 373 children aged 6-23 months were included in this study. One subject was excluded due to insufficient data. Of the sample, $49 \%$ of the subjects were male, and the median age was 14 months (Table 1 ). Water was introduced to about $85 \%$ of the children in the first 3 months of 
Table 1 Characteristics of subjects

\begin{tabular}{lcc}
\hline Characteristics & Total no. of observations & Proportion (\%) \\
\hline Sex (male) & 373 & 49 \\
Age (months) & 373 & $14(6-23)^{\star}$ \\
Introduction of water $<3$ months & 370 & 85 \\
Introduction of CFs $<6$ months & 365 & 62 \\
Colostrum as first food (yes) & 371 & 30 \\
Moment of first breast milk administration & 372 & 47 \\
Before 30 min & 173 & 15 \\
30 min - 1h & 55 & 39 \\
After 1 $\mathrm{h}$ & 144 & $3(1-5)^{\star}$ \\
Number of meals during the day & 279 & 79 \\
Prenatal consultations (yes) & 373 & 66 \\
Vitamin A supplement (yes) & 368 & 92 \\
Mosquito net (yes) & 370 & 58 \\
Zone (living in intervention zone) & 373 & 42 \\
Community & 373 & 21 \\
Galoya & 156 & 34 \\
Podor & 79 & 3 \\
Guede & 125 & 26 \\
Gamadji & 13 & 54 \\
Source of water (from river/pond) & 373 & 90 \\
Family size (>10 individuals) & 373 & 83 \\
Maternal age ( $\geq 20$ yearsr) & 373 & 43 \\
Maternal ethnicity (Halpular) & 373 & 16 \\
Maternal education (literate) & 373 & 66 \\
Maternal employment (employed) & 372 & 17 \\
Recent illness & 373 & 16 \\
Recent diarrhoea & 373 & 20 \\
Weight-for-height Z-score less than - 2 SD & 373 & \\
Height-for-age Z-score less than -2 SD & 373 & \\
Weight-for-age Z-score less than -2 SD & 373 & \\
\hline
\end{tabular}

CFs - complementary foods

* Median value (2.5th to 97.5 th percentile).

life and 62\% were given CFs before 6 months. With regard to growth, 16\% were classified as having wasting and 20\% as having stunting.

There was no statistically significant association between wasting or stunting and introduction of water before 3 months or introduction of CFs before 6 months (Table 2). A significant association was found between wasting and male sex, age, residing in the Guede community, river or pond as the primary source of drinking water and family size greater than 10. A significant association was also found between stunting and age, and river or pond water as not the primary source of drinking water. In post hoc analysis, river or pond as the primary source of drinking water was also associated with recent diarrhoea (odds ratio, 95\% confidence interval, $P$-value: $1.81,1.02-3.22, P=0.04)$. In secondary analysis, linear regression was performed for the main exposures with WHZ and HAZ as continuous variables, and the results were essentially unchanged.

After adjustment for sex, age, Guede community, primary source of drinking water and family size, there was still no association between adverse nutritional status and introduction of water before 3 months (also adjusted for introduction of CFs: WHZ, 1.13, 0.48-2.65, $P=0.8$; HAZ, 0.88, 0.41-1.91, $P=0.8$ ) or introduction of CFs before 6 months (also adjusted for introduction of water: WHZ, 0.79, 0.43-1.46, $P=0.5$; HAZ, 0.85, 0.48-1.51, $P=0.6$ ). Multinomial linear regression revealed no significant interactions by sex, community, zone, source of drinking water, family size, recent illness or recent diarrhoea.

In the qualitative assessment, knowledge of medical recommendations for 6 months of exclusive breastfeeding varied greatly among mothers, and mothers' medical knowledge was customarily superseded by the recommendations of elders. The source of water for most households was determined by availability, but mothers preferred treated tap water over river or pond water for hygienic reasons. Mothers cited disinfectants and head scarf filters as strategies for treating drinking water from open sources. Early introduction of water was often dependent on tradition, while early introduction of CFs was often initiated with the perceived lack of breast milk or with perceived food-seeking behaviours by the infant. Introduction of animal milk often preceded the introduction of solid CFs by several months. The custom of ritual administration of religious water or milk to the newborn on the first day of life was common, and this practice was considered by the mothers (and for our analyses) to be different from introduction of water or CFs.

\section{Discussion}

The prevalence of wasting and stunting in the present study closely reflects previously reported childhood 
Table 2 Unadjusted associations between the subjects' characteristics and their risk for wasting and stunting

\begin{tabular}{|c|c|c|c|c|c|c|}
\hline \multirow[b]{2}{*}{ Unadjusted analyses } & \multicolumn{3}{|c|}{$\begin{array}{c}\text { Wasting } \\
(\text { WHZ less than }-2)\end{array}$} & \multicolumn{3}{|c|}{$\begin{array}{c}\text { Stunting } \\
\text { (HAZ less than }-2)\end{array}$} \\
\hline & OR & $95 \% \mathrm{Cl}$ & $P$ & OR & $95 \% \mathrm{Cl}$ & $P$ \\
\hline Sex (male) & 1.95 & $1.11-3.43$ & 0.02 & 1.66 & $0.98-2.83$ & 0.06 \\
\hline Age (months) & 1.07 & $1.02-1.14$ & 0.01 & 1.09 & $1.03-1.15$ & 0.004 \\
\hline Introduction of water $<3$ months & 0.99 & $0.46-2.14$ & 0.97 & 0.68 & $0.34-1.36$ & 0.3 \\
\hline Introduction of CFs $<6$ months & 0.81 & $0.46-1.42$ & 0.5 & 0.79 & $0.46-1.35$ & 0.4 \\
\hline Colostrum as first food & 0.90 & $0.49-1.66$ & 0.7 & 0.75 & $0.41-1.37$ & 0.3 \\
\hline \multicolumn{7}{|l|}{ First breast milk administration } \\
\hline Before $30 \mathrm{~min}$ & 1.00 & & & 1.00 & & \\
\hline $30 \min -1 \mathrm{~h}$ & 1.71 & $0.61-4.78$ & 0.3 & 1.89 & $0.73-4.85$ & 0.2 \\
\hline After $1 \mathrm{~h}$ & 0.67 & $0.37-1.22$ & 0.2 & 0.94 & $0.54-1.66$ & 0.8 \\
\hline Number of meals during the day & 1.05 & $0.78-1.40$ & 0.8 & 0.95 & $0.72-1.24$ & 0.7 \\
\hline Prenatal consultations (yes) & 0.90 & $0.47-1.74$ & 0.8 & 0.59 & $0.33-1.06$ & 0.08 \\
\hline Vitamin A supplement (yes) & 1.18 & $0.66-2.14$ & 0.6 & 0.79 & $0.45-1.36$ & 0.4 \\
\hline Mosquito net (yes) & 5.74 & $0.77-43.02$ & 0.09 & 1.44 & $0.49-4.30$ & 0.5 \\
\hline Zone (living in intervention zone) & 1.45 & $0.82-2.58$ & 0.2 & 0.83 & $0.49-1.41$ & 0.5 \\
\hline \multicolumn{7}{|l|}{ Community (reference to Galoya) } \\
\hline Galoya & 1.00 & & & 1.00 & & \\
\hline Podor & 0.63 & $0.25-1.54$ & 0.3 & 0.65 & $0.31-1.38$ & 0.3 \\
\hline Guede & 1.94 & $1.05-3.61$ & 0.04 & 0.96 & $0.53-1.74$ & 0.9 \\
\hline Gamadji & 2.86 & $0.81-10.12$ & 0.1 & 0.73 & $0.16-3.48$ & 0.7 \\
\hline Source of water (river/pond) & 2.57 & $1.45-4.56$ & 0.001 & 0.33 & $0.15-0.72$ & 0.005 \\
\hline Family size ( $>10$ individuals) & 2.74 & $1.49-5.06$ & 0.001 & 0.65 & $0.38-1.10$ & 0.1 \\
\hline Maternal age ( $>20$ years) & 1.01 & $0.4-2.54$ & 0.98 & 1.48 & $0.55-3.94$ & 0.4 \\
\hline Maternal ethnicity (Halpular) & 1.48 & $0.67-3.29$ & 0.3 & 1.47 & $0.69-3.15$ & 0.3 \\
\hline Maternal education (literate) & 1.00 & $0.57-1.74$ & 0.99 & 0.80 & $0.47-1.37$ & 0.4 \\
\hline Maternal employment (employed) & 1.24 & $0.6-2.55$ & 0.6 & 0.94 & $0.45-1.97$ & 0.9 \\
\hline Recent illness & 1.69 & $0.91-3.17$ & 0.1 & 0.92 & $0.53-1.60$ & 0.77 \\
\hline Recent diarrhoea & 1.22 & $0.61-2.46$ & 0.6 & 0.59 & $0.27-1.31$ & 0.2 \\
\hline
\end{tabular}

WHZ - weight-for-height Z-score; HAZ - height-for-age Z-score; OR - odds ratio; $\mathrm{Cl}$ - confidence interval; CFs - complementary foods.

malnutrition in Senegal ${ }^{13}$. Our findings suggest that early introduction of water ( $<3$ months) or CFs ( $<6$ months) to an infant's diet does not increase risk for malnutrition from 6 to 23 months of age. Because of the high prevalence of partial breast-feeding in this region $(62 \%$ of children $<6$ months of age), we did not assess the effect of early weaning from breast-feeding, which is known to be associated with increased infant morbidity and mortality in developing countries ${ }^{14}$.

Several cohort studies in developing countries provide mixed reports about the association of early CFs and nutritional status. Simondon and Simondon reported in a cohort of 420 Senegalese infants that introduction of CFs by 2-3 months was associated with significantly lower length-for-age, weight-for-length and arm circumference, and introduction by 4-5 months was associated with decreased linear growth velocity ${ }^{15}$. Brush et al. reported increased growth with later introduction of $\mathrm{CFs}^{16}$, and a large-scale study conducted in Chile reported a higher weight-for-age for exclusively breast-fed infants relative to those fed earlier than 6 months by bottle or solid foods ${ }^{17}$. However, randomised control trials conducted in Honduras reported no difference in weight gain at 4-6 or 9-12 months between children exclusively breast-fed for 6 months and those given CFs of high nutritional and microbiological standards at 4-6 months ${ }^{18,19}$. Several observational studies similarly reported no significant difference in initial weight or length gain between infants exclusively breast-fed for 6 months and those exclusively breast-fed for 4 months followed by mixed breastfeeding ${ }^{20,21}$. A systematic review by Kakuma and Kramer in 2001 suggests that early introduction of CFs has little effect on child growth, but clear negative effects on child health (incidence of diarrhoea) and thus probably on child survival ${ }^{6}$. With regard to introduction of water, Adair et al. demonstrated increased risk of diarrhoea among children fed non-nutritive liquids, an effect mitigated by boiling of the water ${ }^{20}$.

The prevalence of stunting in the present study increased with age, reflecting the presumed accumulation of chronic malnutrition, illness, weaning or other adverse events $^{22}$. The use of tap water, as opposed to water from open sources such as rivers and ponds, was a significant risk factor for stunting. Families utilising tap water may be more prone to live in crowded semi-urban conditions and therefore exposed to additional socio-economic, dietary or non-diarrhoeal infectious disease, such as febrile respiratory infections, for poor growth ${ }^{22}$. The possibility of tap water contamination should also be considered. Whereas mothers preferred tap water for its presumed hygienic superiority, filtering and treatment practices varied widely and were reported mainly among those 
using open water sources, but not among those using tap water. Large family size and drinking from open water sources were both risk factors for wasting, possibly as an indirect result of diarrhoeal disease (as described by the post boc analysis above).

Male sex was found to be associated with a higher prevalence of wasting, as well as a trend for higher prevalence of stunting. Gender differences in nutritional status have not previously been described in young children in Senegal nor in other regions of Africa ${ }^{23,24}$. Previous studies in Senegal reveal no difference in feeding practices between males and females ${ }^{13}$. This gender difference should be investigated in further studies to assess the effect of gender on nutritional status.

There are limitations to the present study. The lack of an association between early introduction of water and CFs and nutritional status may reflect the inclusion of older subjects (18-23 months), as possible negative effects of early feeding may not persist and may be modified by subsequent feeding characteristics and disease events. Unmeasured household characteristics, such as maternal parity, maternal knowledge regarding breast-feeding recommendations, co-habitation with elders, financial status of the household and type of maternal employment, could have introduced unmeasured confounding, but we feel that these factors are unlikely to have altered the main associations. The relatively small sample size is likely to have precluded the ability to detect interactions and to conduct meaningful subgroup analysis. Therefore, we cannot exclude that significant interactions existed but were undetected in the present study. Relatively large confidence intervals for the main associations do not allow us to rule out the possibility of an undetected association (type II error).

Due to the cross-sectional design utilised in this study, there exists the possibility of recall bias. A cross-sectional design is also not suited to control for infant weight and length prior to exposure to water and CFs. Thus the possibility of reverse causality (i.e. infants who grew slowly were more likely to have early introduction of CFs to improve their growth) cannot be excluded. However, this is unlikely to be the case for early introduction of water. Additionally, misclassification of timing of infant feeding may have been present due to inaccurate maternal recall or due to cultural misinterpretation of CFs. For example, some respondents may have considered CFs to include only solid foods and not reported introduction of important CFs such as animal milk. Alternatively, other respondents may have interpreted CFs to include isolated ceremonial feedings administered during the first days of life. Furthermore, CFs were not defined in terms of quantity or nutritional and microbiological standards. Finally, this study may not be generalisable to feeding practices in other regions of Africa, as many of the religious and cultural beliefs of this population, as well as the geographic location and accessibility to clean water and to medical resources, may be unique. Because there exist such limited data on the question of nutritional status following early introduction of water and CFs, we believe that this study's findings are valuable despite these limitations.

Our study also has unique strengths. The cross-sectional study design allowed us to observe a larger sample of children than previous prospective observational and experimental studies. Data were collected within randomly selected households rather than at health clinics, which allowed a more representative sample and equal inclusion of families without access to health care facilities. Unlike previous observational studies, our study analysed the impact of water separately from that of CFs, and controlled for potential confounding by water source and recent medical history. Finally, phase II of this study allowed us to identify some of the reasons for early introduction of water and CFs, and habits regarding water utilisation.

In conclusion, the results of the present study do not show an association between early introduction of water and CFs and malnutrition in infants of northern Senegal and, therefore, do not suggest that these frequent practices increase the risk for malnutrition. These results, however, do not undermine the well-documented benefits of breastfeeding and do not suggest an advantage to early weaning. Additionally, early introduction of CFs may be a risk factor for increased morbidity and decreased survival without adverse effects on growth ${ }^{6}$. Well-known risk factors for malnutrition (family size, access to drinking water and diarrhoeal disease) were confirmed by this study, while apparent gender disparities could lead to further research. The variety of beliefs and behaviours that contribute to infant feeding practices must be carefully examined and subsequently addressed in WHO guidelines. Along with previous studies, our findings highlight the need for larger clinical trials to explore further the role of early feeding in childhood malnutrition, with the goal of developing suitable evidence-based recommendations and interventions to reduce childhood malnutrition in developing countries.

\section{Acknowledgements}

Sources of funding: The study was funded by Fondation Terre des Hommes, the Ford Foundation Grant for the African Health Research Seminar of the University of Pennsylvania, and the Center for Clinical Epidemiology and Biostatistics Summer Research Fellowship of the University of Pennsylvania. M.G. is supported by the Department of Pediatrics of the Centre Hospitalier Universitaire Vaudois of Lausanne, Switzerland. N.S. is supported in part by NIH grant K23 RR16073 and by the Department of Pediatrics of the University of Pennsylvania School of Medicine at The Children's Hospital of Philadelphia. 
Conflict of interest declaration: N.S was a consultant for Wyeth Nutrition and the Dannon Institute and receives research funding from Pfizer.

Authorship responsibilities: N.G. provided substantial contribution to conception and design, acquisition of data, analysis and interpretation of data, and drafting the article. M.G. provided substantial contribution to conception and design, acquisition of data, interpretation of data, and revising the article critically for important intellectual content. N.S. provided substantial contribution to conception and design, analysis and interpretation of data, and revising the article critically for important intellectual content. All authors provided final approval of the version to be published.

Acknowledgements: We would like to thank Dr Cheikh Hanne, Dr Abdulaye Mbaye, Dr Abimbola Lagunju, Mr Aly Diop, Mr Saidou Sall, Dr Papa Kane Gaye and Fondation Terre des Hommes for their assistance and support in the completion of this study.

\section{References}

1 World Health Organization (WHO). Global Strategy for Infant and Young Children Feeding. Geneva: WHO, 2003.

2 Thapar N, Sanderson I. Diarrhoea in children: an interface between developing and developed countries. Lancet 2004; 363: 641-65.

3 Pelletier DL, Frongillo EA, Schroeder DG, Habicht JP. The effects of malnutrition on child mortality in developing countries. Bulletin of the World Health Organization 1995; 73: $443-8$.

4 Rice AL, Sacco L, Hyder A, Black RE. Malnutrition as an underlying cause of childhood deaths associated with infectious diseases in developing countries. Bulletin of the World Health Organization 2000; 78: 1207-21.

5 World Health Organization (WHO). Nutrient Adequacy of Exclusive Breastfeeding for the Term Infant During the First Six Months of Life. Geneva: WHO, 2002.

6 Kramer MS, Kakuma R. The Optimal Duration of Excusive Breastfeeding. Geneva: World Health Organization, 2001.

7 Vaahtera M, Kulmala T, Hietanen A, Ndekha M, Cullinan T, Salin ML, Ashorn P. Breastfeeding and complementary feeding practices in rural Malawi. Acta Paediatrica 2001; 3: $328-32$.

8 Ojofeitimi EO, Olaogun AA, Osokoya AA, Owolabi SP. Infant feeding practices in a deprived environment: a concern for early introduction of water and glucose $\mathrm{D}$ water to neonates. Nutrition and Health 1999; 13: 11-21.

9 Fondation Terre des Hommes. Report of the CAP Survey on the Basic Health and Nutritional State of Children 0 to 36 Months, Pregnant Women, and Nursing Mothers in the
Podor Health District. Saint-Louis, Sénégal: Fondation Terre Des Hommes, 2003 (translation).

10 Médecins Sans Frontières. Nutrition Guidelines. Paris: Médecins Sans Frontières, 1995.

11 World Health Organization (WHO). Management of the Child with a Serious Infection or Severe Malnutrition: Guidelines for Care at the First-referral Level in Developing Countries. Geneva: WHO, 2000.

12 World Health Organization. WHO Global Database on Child Growth and Malnutrition [online]. Available at http:// www.who.int/nutgrowthdb/intro_text.htm. Accessed 12 December 2004.

13 Simondon KB, Simondon F. Mothers prolong breastfeeding of undernourished children in rural Senegal. International Journal of Epidemiology 1998; 27: 490-4.

14 Cunningham AS, Jelliffe DB, Jelliffe EF. Breast-feeding and health in the 1980s: a global epidemiologic review. Journal of Pediatrics 1991; 118: 659-66.

15 Simondon KB, Simondon F. Age at introduction of complementary food and physical growth from 2 to 9 months in rural Senegal. European Journal of Clinical Nutrition 1997; 51: 703-7.

16 Brush G, Harrison GA, Zumrawi FY. A path analysis of some determinants of infant growth in Khartoum. Annals of Human Biology 1993; 20: 381-7.

17 Castillo C, Atalah E, Riumallo J, Castro R. Breast-feeding and the nutritional status of nursing children in Chile. Bulletin of the Pan American Health Organization 1996; 30: 125-33.

18 Cohen RJ, Brown KH, Canahuati J, Rivera LL, Dewey KG. Effects of age of introduction of complementary foods on infant breast milk intake, total energy intake, and growth: a randomized intervention study in Honduras. Lancet 1994; 344: 288-93.

19 Dewey KG, Cohen J, Brown LH, Rivera LL. Age of introduction of complementary foods and growth of term, low-birth-weight, breast-fed infants: a randomized intervention study in Honduras. American Journal of Clinical Nutrition 1999; 69: 679-86.

20 Adair L, Popkin BM, Vanderslice J, Akin J, Guilkey D, Black $\mathrm{R}$, et al. Growth dynamics during the first two years of life: a prospective study in the Philippines. European Journal of Clinical Nutrition 1993; 47: 42-51.

21 Brown KH. The relationship between diarrhoeal prevalence and growth of poor infants varies with their age and usual energy intake. FASEB Journal 1991; 5: A1079 (abstract).

22 Adair LS, Guilkey DK. Age-specific determinants of stunting in Filipino children. Journal of Nutrition 1997; 127: 314-20.

23 Bloss E, Wainaina F, Bailey RC. Prevalence and predictors of underweight, stunting, and wasting among children aged 5 and under in Western Kenya. Journal of Tropical Pediatrics 2004; 50: 260-70.

24 Cartmell E, Natalal H, Francois I, Ferreira MH, Grahnquist L. Nutritional and clinical status of children admitted to the malnutrition ward, Maputo Central Hospital: a comparison of data from 2001 and 1983. Journal of Tropical Pediatrics 2005; 51: 102-5. 